\title{
REMOVABLE SINGULARITIES OF SOLUTIONS OF SYSTEMS OF LINEAR DIFFERENTIAL EQUATIONS
}

\author{
BY TAKAHIRO KAWAI ${ }^{1}$
}

Communicated by S. S. Chern, November 6, 1974

The purpose of this note is to prove theorems on removable singularities of hyperfunction solutions and real analytic solutions of systems of linear differential equations.

It is Ehrenpreis [1] who first showed that any "overdetermined" system of linear differential equations with constant coefficients presents the Hartogs' phenomenon concerning removable singularities of solutions, which is clearly one of the most interesting phenomena in the theory of holomorphic functions of several complex variables. Professor Ehrenpreis also raised a question whether overdetermined systems of linear differential equations with variable coefficients enjoy such a property or not (private communication), and this note gives an affirmative answer to his question in the case of a one point singularity (Theorem 1 below).

We also present the following new phenomenon concerning the extension of real analytic solutions of a system of linear differential equations with variable coefficients:

It may happen that the singularity of a hyperfunction solution is uniquely removable but that an analytic solution cannot be extended as an analytic solution. See Theorem 3 for the precise statement.

It should be emphasized that such a phenomenon can never be observed in the case of systems with constant coefficients.

The details of the proof and the further development of the results of this note shall be given elsewhere.

We use the same notation as Sato-Kawai-Kashiwara [4] unless otherwise stated.

AMS(MOS) subject classifications (1970). Primary 35N10; Secondary 58G99.

Key words and phrases. Removable singularities, overdetermined system, hy perfunctions, real analytic functions.

1 Supported by Miller Institute for Basic Research in Science.

Copyright $\odot$ 1975, American Mathematical Society 
In the sequel $M$ denotes a system of linear differential equations with real analytic coefficients and $M$ denotes the real analytic manifold on which $M$ is defined. We always assume that the system $M$ satisfies the following conditions:

(1) The system $M$ admits a free resolution of length $d$ by the sheaf $D^{f}$ of linear differential operators of finite order.

(2) The system $M$ is purely $d$-dimensional, i.e., $E x t_{D}^{j} f\left(M, D^{f}\right)=0$ holds for $j \neq d \geqslant 2$.

Condition (2) allows us to define the adjoint system $M^{\prime}$ of $M$ by Ext. $t^{d} f\left(M, D^{f}\right) \otimes_{0}\left(\Omega^{n}\right)^{\otimes-1}$. Here $\Omega^{n}$ denotes the sheaf of holomorphic $n$ forms. Making use of $M^{\prime}$, the third condition is stated as follows:

(3) $\operatorname{Ext}_{0}^{j} f\left(M^{\prime}, 0\right)=0$ for $j \geqslant 1$.

Here $O$ denotes the sheaf of holomorphic functions.

See Goldschmidt [2] and references cited there concerning condition (3), which can be reduced to the existence theorem of Cauchy and Kowalevsky outside proper analytic sets.

REMARK 1. Condition (2) asserts that the system $M$ under consideration is an overdetermined system. Though it is possible to argue the extension of hyperfunction solutions of $M$ under the less restrictive condition that $E_{x} t_{D}^{j} f\left(M, D^{f}\right)=0$ for $j=0,1$, we assume (2) in order to avoid technical complexity.

THEOREM 1. Let $M$ be a system of linear differential equations satisfying conditions (1)-(3). Then we have

$$
\operatorname{Ext}_{p t, D}^{j} f(M ; M, B)=0 \text { for } j \neq d .
$$

Here $B$ denotes the sheaf of hyperfunctions and pt denotes a point in $M$.

REMARK 2. One can conclude from Theorem 1 combined with the long exact sequence of relative cohomology groups that any cohomology class in $\operatorname{Ext}_{D f}^{j}(M-p t ; M, B)(j \leqslant d-2)$ can be uniquely extended to a cohomology class in $\operatorname{Ext}_{\delta}^{j} f(M ; M, B)$.

The proof of Theorem 1 can be given by making use of the Serre duality theorem applied to the dual differential complexes constructed by making use of the mutually dual topological vector spaces $B_{p t}$ and $A(p t)$. Here $B_{p t}$ denotes the spaces of hyperfunctions with support in $p t$ and $A(p t)$ denotes the space of real analytic functions defined near $p t$. Conditions (1) and (2) are used to construct the dual differential complexes. As for the Serre duality theorem we refer the reader to Komatsu [3] for example. 
THEOREM 2. Assume that M satisfies conditions (1)-(3). Let $V$ denote the characteristic variety of $M$, and assume the following conditions:

(5) $V$ is regular.

(6) $V \cap \bar{V}$ is regular. Here $\bar{V}$ denotes the complex conjugate of $V$.

(7) $T_{x^{*}}(V) \cap T_{x^{*}}(\bar{V})=T_{x^{*}}(V \cap \bar{V})$ for any point $x^{*}$ in $V \cap \bar{V}$.

(8) The generalized Levi form $L$ of $V$ is of locally constant signature $(p, q)$ on $V \cap \bar{V}$.

If $q$ is always equal to or greater than $r$ with $r \leqslant d-1$, then we have

$$
\operatorname{Ext}_{p t, D}^{j} f(M ; M, A)=0 \text { for } j \leqslant r .
$$

Theorem 3. Assume that M satisfies conditions (1)-(3) and (5)-(8). Assume further that $(p, q)=(d, 0)$ at $(p t, \sqrt{-1} \eta) \in V \cap \bar{V}$. Here $\eta$ denotes a cotangent vector at the point $p t$. Then we have

$$
\operatorname{Ext}_{p t, D f}^{1}(M ; M, A) \neq 0 .
$$

The proofs of Theorems 2 and 3 are given by making use of the long exact sequence of relative cohomology groups and the structure theorem of systems of pseudo-differential equations obtained in Sato-Kawai-Kashiwara [4, Corollary 2.4.2, Chapter III].

REMARK 3. If $p=q=0$ holds identically in Theorem 2 , then $\operatorname{Ext}_{p t, D}^{1} f(M ; M, A)=0$ still holds. It is also the case when $M$ is a system of linear differential equations with constant coefficients which satisfies conditions (1) and (2).

\section{REFERENCES}

1. L. Ehrenpreis, $A$ new proof and an extension of Hartog's theorem, Bull. Amer. Math. Soc. 67 (1961), 507-509. MR 24 \#A1511.

2. H. Goldschmidt, Points singuliers d'un opérateur différentiel analytique, Invent. Math. 9 (1969/70), 165-174. MR 41 \#9278.

3. H. Komatsu, Relative cohomology of sheaves of solutions of differential equations, Lecture Notes in Math., no. 287, Springer, Berlin and New York, 1973, pp. 192261.

4. M. Sato, T. Kawai and M. Kashiwara, Microfunctions and pseudo-differential equations, Lecture Notes in Math., no. 287, Springer-Berlin and New York, 1973, pp. 265-529.

DEPARTMENT OF MATHEMATICS, UNIVERSITY OF CALIFORNIA, BERKELEY, CALIFORNIA 94720

RESEARCH INSTITUTE FOR MATHEMATICAL SCIENCES, KYOTO UNIVERSITY, KYOTO, JAPAN 